ARTIGO ORIGINAL ORIGINAL ARTICLE

\section{Impacto econômico da adoção de denosumabe em pacientes com metástases ósseas ou mieloma múltiplo sob a perspectiva do sistema de saúde privado brasileiro}

\author{
Economic impact of denosumab adoption in cancer \\ patients with bone metastases or multiple myeloma from \\ the Brazilian private healthcare system's perspective \\ Straus Tanaka', Gaurav Suri², Alejandro Arancibia', \\ Danielle do Nascimento', Carlos Magliano³, Guilherme Aratangy' \\ DOI: 10.21115/JBES.v12.n1.p16-22
}

\section{RESUMO}

Objetivo: Estimar o custo por evento relacionado ao esqueleto (ERE) e o impacto econômico anual da adoção de denosumabe em pacientes com metástases ósseas secundárias ao câncer de mama, próstata e outros tumores sólidos ou mieloma múltiplo sob a perspectiva do sistema de saúde privado brasileiro. Métodos: Um modelo econômico foi desenvolvido para comparar os custos relacionados com denosumabe versus ácido zoledrônico na prevenção de EREs. O modelo incluiu os seguintes custos: medicamento, administração, monitoramento e manejo de ERE. O custo anual foi apresentado em reais (BRL) para 100 pacientes. Os custos do manejo de ERE [fratura vertebral (FV), fratura não vertebral (FNV), radiação óssea (RO), cirurgia óssea (CO) e compressão da medula espinhal (CME)] foram estimados a partir dos recursos e procedimentos coletados da revisão de literatura, bases de dados e painel Delphi. Dados coletados dos estudos clínicos randomizados relacionados com cada tipo de tumor na análise e de um estudo prospectivo observacional foram utilizados para estimar a eficácia clínica de denosumabe versus ácido zoledrônico. Resultados: $O$ custo por cada tipo de ERE variou de BRL 27.246 a BRL 28.035 para FV, BRL 18.023 a BRL 18.811 para FNV, BRL 42.750 a BRL 43.538 para RO, BRL 18.023 a BRL 18.811 para CO e BRL 12.472 a BRL 13.260 para CME. A introdução de denosumabe foi estimada em economia anual por 100 pacientes de até BRL 1.072.043,14 para câncer de mama, BRL 1.212.822,79 para outros tumores sólidos, BRL 1.929.660,67 para câncer de próstata e BRL 77.965,07 para mieloma múltiplo. Conclusão: Esta análise sugere que EREs adicionam custos substanciais no manejo de pacientes com metástases ósseas. Dessa forma, o uso de denosumabe pode prevenir e retardar EREs em pacientes com câncer e pode possivelmente levar à redução do impacto econômico associado aos EREs sob a perspectiva dos pagadores de saúde privada brasileira.

\section{Keywords:}

cancer, metastatic breast cancer, multiple myeloma, denosumab, zoledronic acid, bone complication, skeletal related event, economic impact, private healthcare, Brazil

\author{
ABSTRACT \\ Objective: To estimate the cost per SRE and annual economic impact of denosumab adoption in \\ patients with bone metastases (BM) secondary to breast cancer, prostate cancer, other solid tumors \\ or multiple myeloma from the Brazilian private healthcare system's perspective. Methods: An \\ economic model was developed to compare the cost outcomes associated with denosumab instead \\ of zoledronic acid for SRE prevention. The model included the following costs: drug, administration, \\ monitoring and SRE management. Annual costs per 100 patients were reported in 2019 Brazilian

\footnotetext{
Recebido em: 14/01/2020. Aprovado para publicação em: 31/01/2020

1. Amgen, São Paulo, SP, Brasil.

2. Amgen, Uxbridge, Reino Unido.

3. ASAS Valor em Saúde, Rio de Janeiro, RJ, Brasil.

Fontes de financiamento: $O$ estudo foi realizado pela Amgen Brasil.

Conflitos de interesse: Todos os autores são funcionários da Amgen Biotecnologia do Brasil ou da ASAS Valor em Saúde.

Local onde o estudo foi desenvolvido: Amgen Brasil.

Autor correspondente: Straus Tanaka. Rochaverá Corporate Towers (Torre Crystal). Avenida das Nações Unidas, 14171, 22 andar,
} \\ São Paulo, SP, Brasil. CEP: 04794-000. Telefone: +55 (11) 5171-8526. E-mail: syoshito@amgen.com
}


currency (BRL). The SRE management costs (vertebral fracture (VF), non-vertebral fracture (NVF), radiation to bone (RB), surgery to bone (SB) and spinal cord compression (SCC)) were estimated from the resources and procedures collected from literature review, official database, and a Delphi panel. Data collected from randomized clinical trials related to each tumor type in the analysis and from a prospective observational study was used to estimate the clinical efficacy of denosumab vs zoledronic acid. Results: The cost per each type of SREs across all tumors ranged BRL 27,246 - BRL 28,035 for VF, BRL 18,023 - BRL 18,811 for NVF, BRL 42,750 - BRL 43,538 for RB, BRL 18,023 - BRL 18,811 for SB and BRL 12,472 - BRL 13,260 for SCC. The introduction of denosumab was estimated to result in annual savings per 100 patients of up to BRL 1,072,043.14 for breast cancer, BRL 1,212,822.79 for other solid tumors, BRL 1,929,660.67 for prostate cancer and BRL 77,965.07 for multiple myeloma. Conclusion: This analysis suggests that SREs add substantial costs to the management of patients with bone metastases. In this way, the use of denosumab would prevent and delay SREs in cancer patients and might possibly lead to reduce the economic burden associated with SREs, borne by Brazilian private healthcare payers.

\section{Introdução}

Nas últimas décadas, o melhor conhecimento da biologia da doença, assim como avanços em screening, diagnóstico e o surgimento de novas abordagens terapêuticas cada vez mais personalizadas, têm melhorado substancialmente o prognóstico de alguns tipos de câncer, como o câncer de mama, próstata e pulmão (Ganz, 2015; Kim et al., 2012). Houve notável declínio na mortalidade por câncer nas últimas duas décadas: a taxa de mortalidade por câncer de pulmão caiu 48\% no sexo masculino entre 1990 e 2016 e $23 \%$ no sexo feminino entre 2002 e 2016, enquanto a taxa de câncer de mama caiu 40\% de 1989 a 2016 e a taxa de mortalidade por câncer de próstata caiu 51\% de 1993 a 2016 (Siegel et al., 2019). A melhora no prognóstico também incluiu pacientes com doença metastática ao diagnóstico, com melhora das taxas de sobrevida em cinco anos para câncer de mama e próstata metastático (Siegel et al., 2014, 2019). Isso leva a um número crescente de pacientes com doença metastática que vivem por mais tempo. Independentemente de o paciente ser recém-diagnosticado no estágio IV ou ser paciente com câncer metastático como resultado da progressão da doença, ambos apresentam maior risco de sofrerem complicações do câncer a longo prazo. A metástase óssea é a complicação mais frequente do câncer, ocorrendo em até $75 \%$ dos pacientes com câncer de mama ou próstata, aproximadamente 30\% a 40\% dos pacientes com câncer de pulmão e 20\% a 32\% em pacientes com câncer renal (Coleman, 2001, 2006; Saad et al., 2006; Woodward et al., 2011; Yucel et al., 2015). A doença óssea metastática não apenas acarreta dor, prejuízo da qualidade de vida e outras complicações onerosas conhecidas coletivamente como eventos relacionados ao esqueleto (EREs: fratura patológica, compressão da medula espinhal, cirurgia óssea e radiação óssea) (Costa et al., 2008; Saad et al., 2006), como também aumenta o risco de morte (Saad et al., 2007b; Sathiakumar et al., 2011). A doença óssea metastática aumenta em quase sete vezes o risco de morte em pacientes com câncer de próstata, enquanto pacientes com doença óssea metastática e EREs têm um risco de mortalidade 10 ve- zes maior em comparação com aqueles sem acometimento esquelético (Sathiakumar et al., 2011).

Os EREs também podem incidir em pacientes com neoplasias hematológicas que afetam o osso, como o mieloma múltiplo (MM) (Terpos et al., 2019). Estima-se que pacientes com metástase óssea, se não tratados, experimentam um ERE em 70\% a 95\% dos pacientes com MM (Terpos et al., 2019; von Moos et al., 2008), 40\% a 50\% dos pacientes com câncer de próstata (McDougall et al., 2016) e cerca de 50\% dos pacientes com câncer de mama (Domchek et al., 2000; Oster et al., 2013), levando a uma carga substancial para o paciente e para o sistema de saúde (Body et al., 2016; McDougall et al., 2016). Quase metade (46,3\%) da população de tumores sólidos com metástase óssea sem tratamento apresenta EREs dentro de um ano após o primeiro diagnóstico de metástase óssea (Body et al., 2016; McDougall et al., 2016). A maioria dos pacientes com metástases ósseas do estudo de câncer de pulmão de células não pequenas (CPCNP) experimentou um ERE dentro dos primeiros cinco meses de acompanhamento (Coleman, 2001).

Agentes modificadores ósseos como os bisfosfonatos e denosumabe (inibidor de RANK-L) podem reduzir as chances de desenvolvimento do primeiro e dos subsequentes EREs em pacientes com câncer (Fizazi et al., 2011; Henry et al., 2014; Saad et al., 2002; Smith et al., 2009).

O denosumabe foi significantemente superior ao ácido zoledrônico (AZ) em retardar o acontecimento do primeiro ERE, assim como o risco de múltiplos EREs em pacientes com câncer de mama, próstata e outros tumores sólidos (Fizazi et al., 2011; Henry et al., 2014; Lipton \& Balakumaran, 2012; Stopeck et al., 2010). O denosumabe também retardou significantemente o desenvolvimento de dor moderada a grave, assim como o agravamento da escala de dor. A estimativa mediana de curva Kaplan-Meier (95\% IC) para um aumento $\geq 2$ pontos na BPI-SF (Avaliação Brief Pain Inventory-Short Form de 11 pontos) foi de 8,2 [intervalo de confiança (IC) 5,6; 9,6] meses para o denosumabe e de 4,8 (IC 3,7; 7,5) meses para o AZ [hazard ratio (HR): 0,77; IC de 95\%: 0,61 a 0,96, $p=0,021$ ]. Entre os pacientes com pouco ou nenhum uso de analgésicos antes do início do 
estudo, o uso de opioides fortes em cada avaliação foi menor para os pacientes em tratamento com denosumabe do que para pacientes em tratamento com AZ (Henry et al., 2014).

Em um cenário econômico desafiador como no Brasil, o impacto econômico dos EREs em pacientes com tumores sólidos ou MM pode ter um impacto substancial nos sistemas de saúde público e privado. Dessa maneira, a avaliação econômica de agentes modificadores ósseos com o objetivo de maximizar a alocação de recursos nesses pacientes apresenta extrema relevância. Estudos recentes demonstram que denosumabe é uma opção de tratamento mais custo-efetiva para a prevenção de EREs em pacientes com câncer de próstata, mama e outros tumores sólidos quando comparada ao AZ (Cristino et al., 2017; Terpos et al., 2019). Entretanto, o benefício econômico de denosumabe no lugar do AZ no Brasil continua incerto.

\section{Métodos}

Um modelo econômico foi desenvolvido para estimar: (1) o impacto econômico associado aos eventos relacionados ao esqueleto em pacientes com metástases ósseas secundárias ao câncer de mama, próstata, tumores sólidos e em pacientes com MM; (2) o impacto econômico da adoção de denosumabe quando comparado ao AZ na prevenção de EREs sob a perspectiva do sistema de saúde suplementar brasileiro.

\section{Avaliação econômica}

Para a análise de custo no sistema suplementar de saúde brasileiro, foram incluídos os seguintes componentes: aquisição e administração dos medicamentos Xgeva ${ }^{\circledast}$ e Zometa ${ }^{\circledast}$ conforme as informações de bula; monitoramento (exames laboratoriais e de imagem, e consultas médicas); custo de manejo de ERE. Os preços dos medicamentos foram obtidos a partir da lista oficial de preços de medicamentos publicada em setembro de 2019, da Câmara de Regulação do Mercado de Medicamentos (CMED) (Tabela 1).
Para o produto Zometa ${ }^{\circledast}$, foi utilizado o preço fábrica (PF) com $0 \%$ de ICMS, enquanto para o produto Xgeva ${ }^{\oplus}$ foi utilizado o PF com 18\% de ICMS. A diferença de impostos entre os produtos foi considerada na análise, visto que atualmente o princípio ativo AZ é isento do ICMS, de acordo com o Convênio no 140/2001, que concede isenção do ICMS nas operações com medicamentos.

O custo de administração, monitoramento e manejo de EREs foi baseado em uma revisão de literatura e em um painel Delphi que incluiu 24 médicos brasileiros de diferentes especialidades [oncologia (7), radioterapia (4), hematologia (6) e ortopedia (7)], a fim de identificar os procedimentos e recursos mais utilizados no manejo de cada tipo de ERE e garantir que fossem incluídos na análise.

Houve uma ligeira diferença no manejo de EREs adotado para os pacientes com outros tumores sólidos em comparação aos demais pacientes (pacientes com câncer de mama, próstata e MM), que se traduziu em uma pequena diferença no custo do manejo de EREs (Tabela 2). Essa diferença foi possivelmente resultado de uma menor frequência de exames de monitoramento (laboratoriais, de imagem e consultas médicas) ao longo de um ano, em consequência da menor expectativa de sobrevida esperada para esses pacientes com outros tumores sólidos.

Todos os procedimentos e recursos identificados foram precificados de acordo com a lista de Classificação Brasileira Hierarquizada de Procedimentos Médicos (CBHPM 2018 e Simpro) e comparados com os dados de requisição médica (health claims) de prestadores de saúde, a fim de aumentar a fidedignidade dos custos estimados a partir dos dados do painel Delphi. Os custos estimados se apresentaram em acordo ou abaixo dos valores encontrados nas requisições médicas de prestadores de saúde. Assim, para a análise, optou-se por uma abordagem conservadora e foram considerados os valores estimados a partir dos dados do painel Delphi.

A pesquisa com especialistas também identificou que os custos para a administração intravenosa (IV) e subcutânea

Tabela 1. Preço dos medicamentos Xgeva ${ }^{\oplus}$ e Zometa ${ }^{\oplus}$ - Lista de preços da CMED (setembro 2019)

\begin{tabular}{lcccc}
\hline Substância & Produto & Apresentação & PF 0\% & PF 18\% \\
\hline Ácido zoledrônico & Zometa $^{\oplus}$ & $\begin{array}{c}\text { 4 MG SOL INJ CT FA PLAS } \\
\text { TRANS X 100 ML }\end{array}$ & $1.350,18$ & - \\
\hline Denosumabe & Xgeva $^{\oplus}$ & $\begin{array}{c}\text { 120 MG SOL IN CT FA VD } \\
\text { TRANS X 1,7 ML }\end{array}$ & $1.205,83$ & $1.470,52$ \\
\hline
\end{tabular}

Tabela 2. Custo do manejo de eventos relacionados ao esqueleto de acordo com a doença primária

\begin{tabular}{lcccc}
\hline ERE & Câncer de mama & Câncer de próstata & Outros tumores sólidos & Mieloma múltiplo \\
\hline Fratura vertebral & BRL 28.034,54 & BRL 28.034,54 & BRL 27.246,15 & BRL 28.034,54 \\
\hline Fratura não vertebral & BRL 18.810,98 & BRL 18.810,98 & BRL 18.022,59 & BRL 18.810,98 \\
\hline Radiação óssea & BRL 43.537,95 & BRL 43.537,95 & BRL 42.749,56 & BRL 43.537,95 \\
\hline Cirurgia óssea & BRL 18.810,98 & BRL 18.810,98 & BRL 18.022,59 & BRL 18.810,98 \\
\hline Compressão medular & BRL 13.260,22 & BRL 13.260,22 & BRL 12.471,83 & BRL 13.260,22 \\
\hline
\end{tabular}


(SC), incluindo os insumos utilizados e honorários médicos, foram de BRL 161,56 para AZ e de 29,31 para denosumabe.

\section{Frequência de ERE}

A incidência anual de EREs (fratura patológica, radiação óssea, cirurgia óssea ou compressão medular) para pacientes com metástase óssea tratados com denosumabe e AZ foi estimada a partir dos estudos clínicos randomizados relacionados com cada tipo de tumor incluído na análise (Fizazi et al., 2011; Henry et al., 2011; Raje et al., 2018; Stopeck et al., 2010) e ajustada pelos dados do estudo prospectivo observacional (Hechmati et al., 2013), que incluiu 631 pacientes com tumores sólidos ou MM e metástase óssea de quatro países diferentes da Europa (Alemanha, Itália, Espanha e Inglaterra) (Tabelas 3 e 4). A incidência observada foi 2,8 vezes maior que os valores obtidos nos estudos clínicos randomizados.

\section{Resultados}

O custo do manejo dos EREs foi maior para pacientes com metástase óssea secundária ao câncer de próstata (Tabela 5), resultado da maior proporção de procedimentos de maior custo prescritos (i.e., a radiação óssea; Tabela 4).
Os custos de medicação e administração de AZ por ano e por paciente foram de BRL 16.122 e BRL 2.107, respectivamente, para todos os tumores. O custo do manejo de EREs nos pacientes tratados com AZ variou de BRL 45.056 a BRL 97.349, de acordo com o tipo de tumor (Figura 1). Para o denosumabe, os custos anuais totais com o medicamento e administração por paciente corresponderam a BRL 19.182 e BRL 382, respectivamente, para todos os tipos de tumores, enquanto os custos de manejo de ERE variaram de BRL 41.173 a BRL 76.717, de acordo com o tipo de tumor (Figura 2).

$\mathrm{Na}$ análise dos custos anuais totais para a prevenção e o manejo de ERE considerando o AZ, os custos de manejo dos EREs corresponderam a 84\% do custo anual total para o câncer de próstata, 83\% para outros tumores sólidos, 74\% para o câncer de mama e $71 \%$ para o MM, enquanto para o denosumabe os custos de manejo do ERE representaram $80 \%$ do custo anual do câncer de próstata, 79\% para outros tumores sólidos, 69\% para o MM e 68\% para o câncer de mama (Figuras 1 e 2).

Em um cenário com 100 pacientes, o uso de denosumabe como agente modificador ósseo pode levar a uma economia de BRL 1.929.660,67 para câncer de próstata, de BRL 1.212.822,79 para outros tumores sólidos, de BRL 1.072.043,14 para câncer de mama e de BRL 77.965,07 para MM (Figura 3).

Tabela 3. Incidência anual de ERE por tipo de tumor

\begin{tabular}{lcccc}
\hline Medicamento & Câncer de próstata & Câncer de mama & Outros tumores sólidos & Mieloma múltiplo \\
\hline Denosumabe & 2,12 & 1,39 & 2,25 & 1,69 \\
\hline Ácido zoledrônico Q3W/Q4W & 2,69 & 1,79 & 2,66 & 1,77 \\
\hline
\end{tabular}

Tabela 4. Distribuição de ERE de acordo com o tipo de tumor

\begin{tabular}{lcccc}
\hline ERE & Câncer de próstata & Câncer de mama & Outros tumores sólidos & Mieloma múltiplo \\
\hline Fratura vertebral & $15 \%$ & $24 \%$ & $14 \%$ & $42 \%$ \\
\hline Fratura não vertebral & $12 \%$ & $34 \%$ & $18 \%$ & $39 \%$ \\
\hline Radiação óssea & $66 \%$ & $35 \%$ & $58 \%$ & $11 \%$ \\
\hline Cirurgia óssea & $2 \%$ & $5 \%$ & $6 \%$ & $7 \%$ \\
\hline Compressão medular & $6 \%$ & $2 \%$ & $5 \%$ & $1 \%$ \\
\hline
\end{tabular}

Tabela 5. Custo do manejo de ERE de acordo com o tipo de tumor

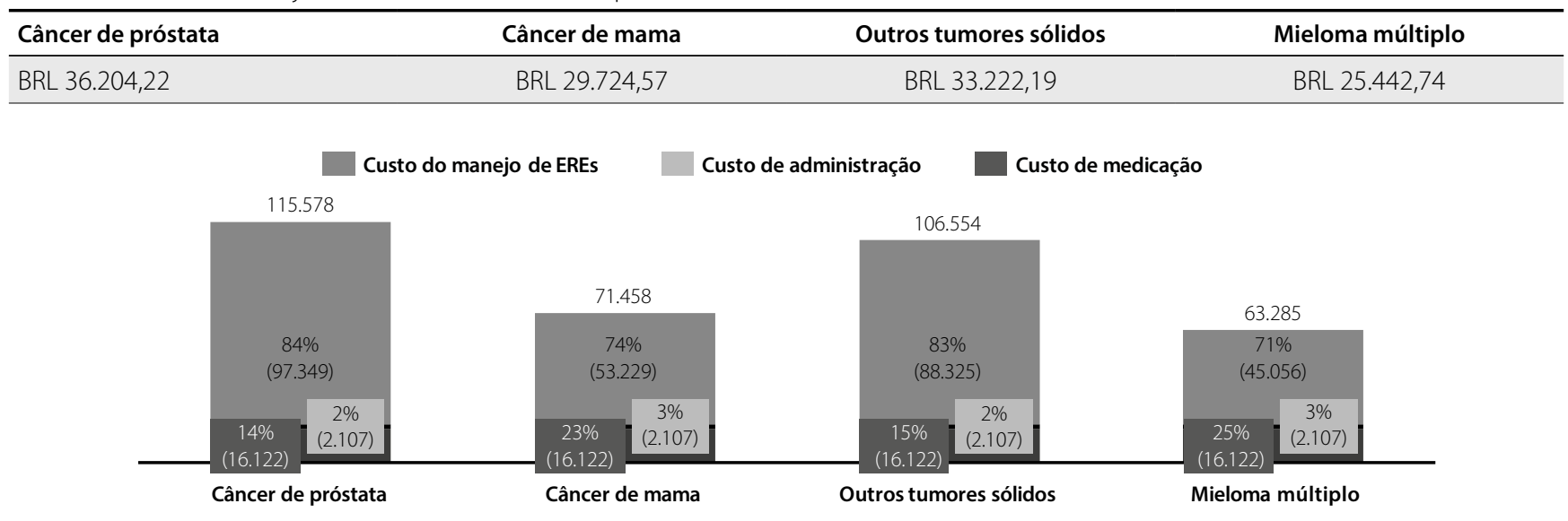

Figura 1. Custo geral (BRL) em 1 ano da prevenção e manejo de EREs com ácido zoledrônico. 


\section{Discussão}

Como resultado dos avanços científicos nas últimas décadas, os prognósticos melhoraram substancialmente para pacientes com câncer metastático, levando a uma ampliação no escopo de abordagem, considerando-se não apenas o manejo do câncer primário, mas também as complicações de médio a longo prazo que esses pacientes estariam mais propensos a sofrer. A metástase óssea é a complicação mais comum do câncer e, se não tratada, o risco de desenvolver um ERE pode chegar a 50\%, dependendo do tipo de câncer (Bhowmik et al., 2019). Além disso, uma vez que um paciente experimenta o primeiro ERE, a probabilidade de experimentar um segundo ERE aumenta consideravelmente e o tempo para o desenvolvimento de EREs subsequentes é reduzido (Saad et al., 2007a).

Os EREs impõem não apenas grande impacto físico e na qualidade de vida aos pacientes, mas também estão associados a uma carga econômica substancial para o sistema de saúde. No cenário econômico atual brasileiro é crucial avaliar o impacto dos EREs no sistema de saúde. A administração de agentes modificadores ósseos como bisfosfonatos e denosumabe antes da ocorrência de um ERE tem sido associada a prevenção ou retardo na ocorrência de EREs, prevenção ou redução da dor óssea e melhorias tanto na qualidade de vida como no status de desempenho do paciente (Henry et al., 2014). No entanto, ao considerar o ônus econômico geral dos EREs, é vital levar em conta não apenas o custo do medicamento bruto, mas também os custos do manejo de EREs, a fim de obter uma compreensão mais ampla da alocação de recursos. Os recursos gastos no manejo de EREs podem impor um grande impacto econômico ao sistema de saúde, chegando a $84 \%$ dos custos totais relacionados à prevenção e ao manejo de EREs. Ao comparar os custos anuais globais para a prevenção e o tratamento de EREs, o AZ apresentou custos mais altos em comparação ao denosumabe para todos os tumores sólidos (câncer de próstata, câncer de mama e outros tumores sólidos). Além disso, a cada 100 pacientes, o uso de denosumabe para a prevenção de ERE pode contribuir com uma economia anual de BRL 1.929.660,67 para câncer de próstata, de BRL 1.072.043,14 para câncer de mama, de BRL 1.212.822,79 para outros tumores sólidos e de BRL $77.965,07$ para MM. Essa economia de recursos não está relacionada apenas à maior eficácia do denosumabe no retardo dos EREs, mas também ao maior custo da administração IV do $A Z$, quando comparado ao da administração SC de denosumabe. Esses resultados estão de acordo com outros estudos que objetivaram medir a carga econômica dos EREs, apesar das diferenças econômicas em todos os países.

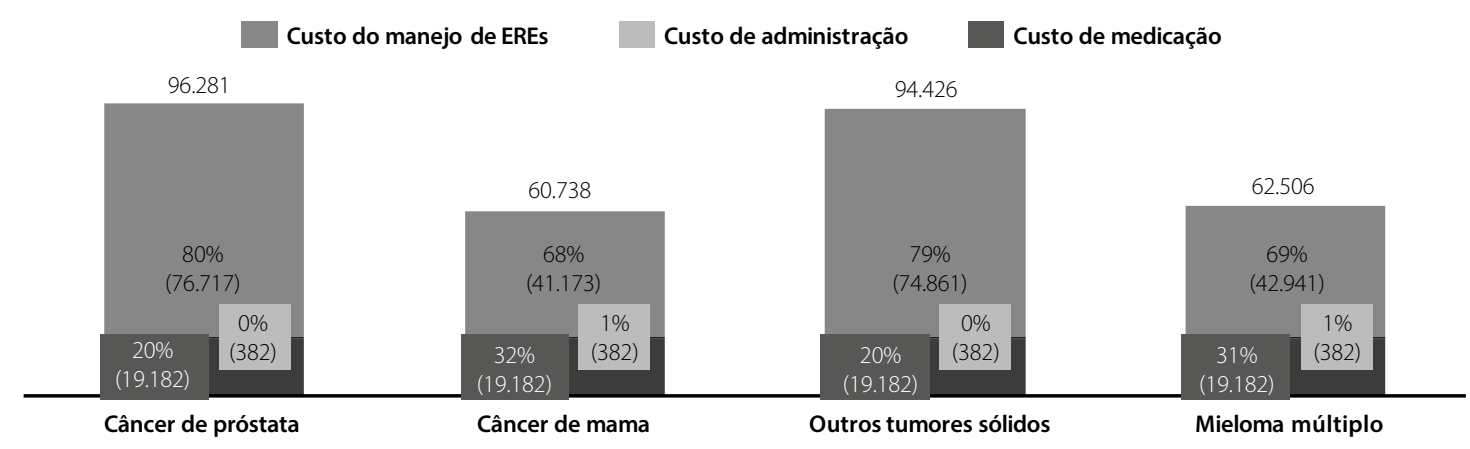

Figura 2. Custo geral (BRL) em 1 ano da prevenção e manejo de EREs com denosumabe.

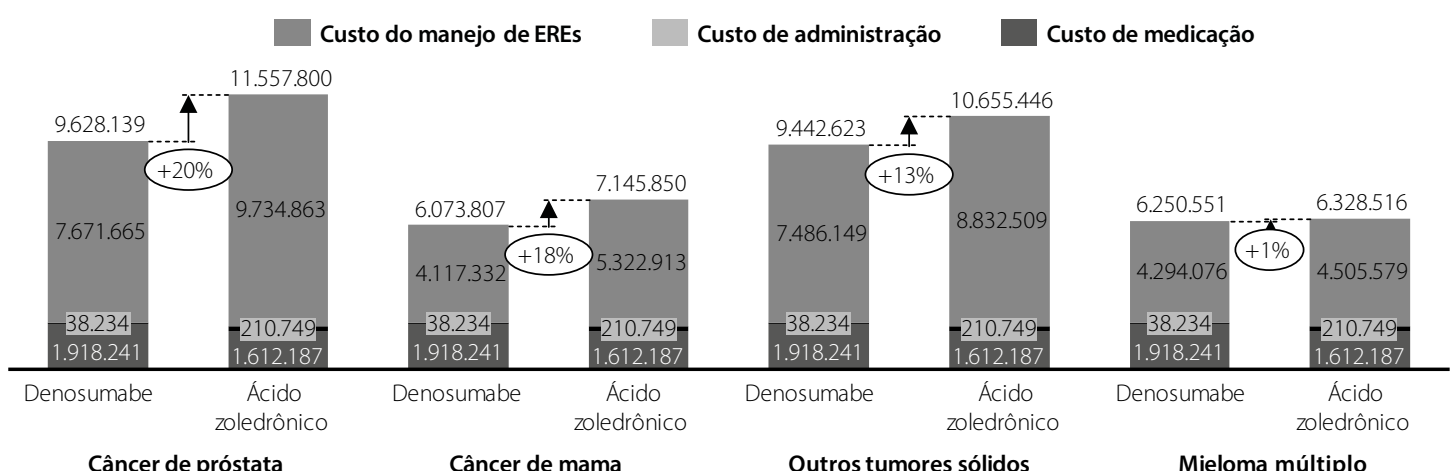

Figura 3. Custo geral (BRL) em 1 ano de 100 pacientes para prevenção e manejo de EREs com ácido zoledrônico e denosumabe. 
Estudos com dados de reembolso médico nos Estados Unidos mostraram que o desenvolvimento de EREs em pacientes com MM foi associado a um aumento anual de 75\% nos custos diretos de saúde, quando comparados a pacientes sem ERE (Bhowmik et al., 2018), e a um incremento de US\$ 67.257 em custos, quando comparados a pacientes sem ERE, para tumores sólidos (Bhowmik et al., 2019).

A presente análise não considerou fatores que também poderiam interferir na análise de custos, como a qualidade de vida e o risco de nefrotoxicidade do uso de AZ. O impacto clínico e econômico acumulado devido ao risco de nefrotoxicidade ainda não está bem estabelecido na literatura, e a inclusão desse critério na análise poderia adicionar incertezas nos resultados finais.

No entanto, dados de estudos clínicos sugerem que os pacientes oncológicos são de alguma forma afetados ou têm limitações de tratamento durante o curso do tratamento devido à insuficiência renal. Entre os pacientes que foram tratados com um medicamento anticâncer, estima-se que 79,9\% receberam pelo menos um medicamento que exigia um ajuste da dose ou para o qual não havia dados para uso em pacientes com insuficiência renal e 80,1\% receberam pelo menos uma droga potencialmente nefrotóxica (Lameire, 2014). Além disso, foi relatado que aproximadamente $60 \%$ dos pacientes apresentam ou desenvolvem disfunção renal durante o curso do MM (Kanellias et al., 2018). Considerando o risco de nefrotoxicidade em pacientes com câncer, o denosumabe deve ser considerado uma opção preferida de modificação óssea, já que pode ser prescrito independentemente da função renal, não necessita de ajuste da dose e raramente resulta em insuficiência renal, enquanto os bifosfonatos devem ser usados com cautela em pacientes com insuficiência renal (clearance de creatinina $<30 \mathrm{~mL}$ / min) e devem ter a dose ajustada de acordo com a função renal (clearance de creatinina $<60 \mathrm{~mL} / \mathrm{min}$ ) (Bagratuni et al., 2018).

\section{Conclusão}

O uso de AZ na prevenção de ERE em pacientes com diagnóstico de metástase óssea secundária a câncer de próstata, câncer de mama, outros tumores sólidos ou MM possivelmente está associado a custos anuais substancialmente mais altos em comparação ao uso de denosumabe, na perspectiva do sistema de saúde privado brasileiro.

\section{Referências bibliográficas}

Bagratuni T, Ntanasis-Stathopoulos I, Gavriatopoulou M, MavrianouKoutsoukou N, Liacos C, Patseas D, et al. Detection of MYD88 and CXCR4 mutations in cell-free DNA of patients with IgM monoclonal gammopathies. Leukemia. 2018;32(12):2617-25.

Bhowmik D, Hines DM, Intorcia M, Wade RL. Economic burden of skeletalrelated events in patients with multiple myeloma: analysis of US commercial claims database. J Med Econ. 2018;21(6):622-8.
Bhowmik D, Song X, Intorcia M, Gray S, Shi N. Examination of burden of skeletal-related events in patients naive to denosumab and intravenous bisphosphonate therapy in bone metastases from solid tumors population. Curr Med Res Opin. 2019;35(3):513-23.

Body JJ, Pereira J, Sleeboom H, Maniadakis N, Terpos E, Acklin YP, et al. Health resource utilization associated with skeletal-related events: results from a retrospective European study. Eur J Health Econ. 2016;17(6):711-21.

Coleman RE. Metastatic bone disease: clinical features, pathophysiology and treatment strategies. Cancer Treat Rev. 2001;27(3):165-76.

Coleman RE. Clinical features of metastatic bone disease and risk of skeletal morbidity. Clin Cancer Res. 2006;12(20 Pt 2):6243s-9s.

Costa L, Badia X, Chow E, Lipton A, Wardley A. Impact of skeletal complications on patients' quality of life, mobility, and functional independence. Support Care Cancer. 2008;16(8):879-89.

Cristino J, Finek J, Jandova P, Kolek M, Pásztor B, Giannopoulou C, et al. Cost-effectiveness of denosumab versus zoledronic acid for preventing skeletal-related events in the Czech Republic. J Med Econ. 2017;20(8):799-812.

Domchek SM, Younger J, Finkelstein DM, Seiden MV. Predictors of skeletal complications in patients with metastatic breast carcinoma. Cancer. 2000;89(2):363-8.

Fizazi K, Carducci M, Smith M, Damião R, Brown J, Karsh L, et al. Denosumab versus zoledronic acid for treatment of bone metastases in men with castration-resistant prostate cancer: a randomised, double-blind study. Lancet. 2011;377(9768):813-22.

Ganz P. Improving outcomes for breast cancer survivors: perspectives on research challenges and opportunities. In: Ganz P, ed. Advances in experimental medicine and biology. New York/Dordrecht/London: Springer/Cham/Heidelberg; 2015. v. 862

Hechmati G, Cure S, Gouépo A, Hoefeler H, Lorusso V, Lüftner D, et al. Cost of skeletal-related events in European patients with solid tumours and bone metastases: data from a prospective multinational observational study. J Med Econ. 2013;16(5):691-700.

Henry D, Vadhan-Raj S, Hirsh V, von Moos R, Hungria V, Costa L, et al. Delaying skeletal-related events in a randomized phase 3 study of denosumab versus zoledronic acid in patients with advanced cancer: an analysis of data from patients with solid tumors. Support Care Cancer. 2014;22(3):679-87.

Henry DH, Costa L, Goldwasser F, Hirsh V, Hungria V, Prausova J, et al. Randomized, double-blind study of denosumab versus zoledronic acid in the treatment of bone metastases in patients with advanced cancer (excluding breast and prostate cancer) or multiple myeloma. J Clin Oncol. 2011;29(9):1125-32.

Kanellias N, Gavriatopoulou M, Terpos E, Dimopoulos MA. Management of multiple myeloma bone disease: impact of treatment on renal function. Expert Rev Hematol. 2018;11(11):881-8.

Kim MM, Hoffman KE, Levy LB, Frank SJ, Pugh TJ, Choi S, et al. Improvement in prostate cancer survival over time: a 20 -year analysis. Cancer J. 2012;18(1):1-8.

Lameire N. Nephrotoxicity of recent anti-cancer agents. Clin Kidney J. 2014;7(1):11-22.

Lipton A, Balakumaran A. Denosumab for the treatment of cancer therapyinduced bone loss and prevention of skeletal-related events in patients with solid tumors. Expert Rev Clin Pharmacol. 2012;5(4):359-71.

McDougall JA, Bansal A, Goulart BH, McCune JS, Karnopp A, Fedorenko C, et al. The Clinical and Economic Impacts of Skeletal-Related Events Among Medicare Enrollees With Prostate Cancer Metastatic to Bone. Oncologist. 2016;21(3):320-6.

Oster G, Lamerato L, Glass AG, Richert-Boe KE, Lopez A, Chung K, et al. Natural history of skeletal-related events in patients with breast, lung, or 
prostate cancer and metastases to bone: a 15-year study in two large US health systems. Support Care Cancer. 2013;21(12):3279-86.

Raje N, Terpos E, Willenbacher W, Shimizu K, García-Sanz R, Durie B, et al. Denosumab versus zoledronic acid in bone disease treatment of newly diagnosed multiple myeloma: an international, double-blind, double-dummy, randomised, controlled, phase 3 study. Lancet Oncol. 2018;19(3):370-81.

Saad F, Chen YM, Gleason DM, Chin J. Continuing benefit of zoledronic acid in preventing skeletal complications in patients with bone metastases. Clin Genitourin Cancer. 2007a;5(6):390-6.

Saad F, Clarke N, Colombel M. Natural history and treatment of bone complications in prostate cancer. Eur Urol. 2006;49(3):429-40.

Saad F, Gleason DM, Murray R, Tchekmedyian S, Venner P, Lacombe L, et al.; Zoledronic Acid Prostate Cancer Study Group. A randomized, placebocontrolled trial of zoledronic acid in patients with hormone-refractory metastatic prostate carcinoma. J Natl Cancer Inst. 2002;94(19):1458-68.

Saad F, Lipton A, Cook R, Chen YM, Smith M, Coleman R. Pathologic fractures correlate with reduced survival in patients with malignant bone disease. Cancer. 2007b;110(8):1860-7.

Sathiakumar N, Delzell E, Morrisey MA, Falkson C, Yong M, Chia V, et al. Mortality following bone metastasis and skeletal-related events among men with prostate cancer: a population-based analysis of US Medicare beneficiaries, 1999-2006. Prostate Cancer Prostatic Dis. 2011;14(2):177-83.

Siegel R, Ma J, Zou Z, Jemal A. Cancer statistics, 2014. CA Cancer J Clin. 2014;64(1):9-29.
Siegel RL, Miller KD, Jemal A. Cancer statistics, 2019. CA Cancer J Clin. 2019;69(1):7-34.

Smith MR, Egerdie B, Hernández Toriz N, Feldman R, Tammela TL, Saad F, et al.; Denosumab HALT Prostate Cancer Study Group. Denosumab in men receiving androgen-deprivation therapy for prostate cancer. N Engl J Med. 2009;361(8):745-55.

Stopeck AT, Lipton A, Body JJ, Steger GG, Tonkin K, de Boer RH, et al. Denosumab compared with zoledronic acid for the treatment of bone metastases in patients with advanced breast cancer: a randomized, double-blind study. J Clin Oncol. 2010;28(35):5132-9.

Terpos E, Jamotte A, Christodoulopoulou A, Campioni M, Bhowmik D, Kennedy $L$, et al. A cost-effectiveness analysis of denosumab for the prevention of skeletal-related events in patients with multiple myeloma in four European countries: Austria, Belgium, Greece, and Italy. J Med Econ. 2019;22(8):766-76.

von Moos R, Strasser F, Gillessen S, Zaugg K. Metastatic bone pain: treatment options with an emphasis on bisphosphonates. Support Care Cancer. 2008;16(10):1105-15.

Woodward E, Jagdev S, McParland L, Clark K, Gregory W, Newsham A, et al. Skeletal complications and survival in renal cancer patients with bone metastases. Bone. 2011;48(1):160-6.

Yucel B, Celasun MG, Oztoprak B, Hasbek Z, Bahar S, Kacan T, et al. The negative prognostic impact of bone metastasis with a tumor mass. Clinics (Sao Paulo). 2015;70(8):535-40. 